Cahiers $d u$ MONDE RUSSE

\section{Cahiers du monde russe}

Russie - Empire russe - Union soviétique et États indépendants

$51 / 4 \mid 2010$

Sciences humaines et sociales en Russie à l'Âge d'argent

\title{
David Shneer, Through Soviet Jewish Eyes
}

\section{Nathalie Moine}

\section{(2) OpenEdition}

Journals

Édition électronique

URL : https://journals.openedition.org/monderusse/7450

DOI : $10.4000 /$ monderusse. 7450

ISSN : $1777-5388$

Éditeur

Éditions de l'EHESS

Édition imprimée

Date de publication : 25 novembre 2010

Pagination : 824-827

ISBN : 978-2-7132-2316-7

ISSN : $1252-6576$

Référence électronique

Nathalie Moine, "David Shneer, Through Soviet Jewish Eyes », Cahiers du monde russe [En ligne], 51/4 | 2010, mis en ligne le 09 décembre 2011, consulté le 03 septembre 2022. URL : http:// journals.openedition.org/monderusse/7450; DOI : https://doi.org/10.4000/monderusse.7450

Ce document a été généré automatiquement le 3 septembre 2022

Tous droits réservés 


\title{
David Shneer, Through Soviet Jewish Eyes
}

\author{
Nathalie Moine
}

\section{RÉFÉRENCE}

David SHNEER, Through Soviet Jewish Eyes. Photography, War and the Holocaust.

New Brunswick, NJ : Rutgers University Press, 2011, 283 p.

1 Spécialiste de la culture yiddish des débuts de l'URSS, David Shneer signe avec cet ouvrage une passionnante histoire des photographes reporters juifs soviétiques qui participèrent à la couverture de la guerre, et notamment de l'Holocauste en Union soviétique. L'étude dépasse largement le cadre de la guerre pour offrir un portrait de groupe qui suit les protagonistes depuis leur lieu d'origine, souvent des villes du sud et de l'ouest de l'ancien Empire russe, jusqu'aux emplacements des massacres découverts par l'Armée rouge, en passant par leurs débuts professionnels dans la capitale du jeune État soviétique de l'entre-deux guerres. David Shneer rappelle que la photographie, dès avant la révolution, a attiré des juifs de l'Empire, puisqu'il s'agissait d'un medium nouveau, donc dépourvu d'une institutionnalisation discriminante, et nécessitant le sens de l'entreprise.

Cependant, la révolution marque un tournant: ce sont majoritairement les juifs qui construisent une nouvelle forme d'expression au service du jeune État bolchevik, le photojournalisme. Pour ces jeunes gens, essentiellement des hommes, la photographie est un vecteur de promotion sociale et de rupture avec le milieu familial traditionnel. Plusieurs déjudaïsent leurs noms et mettent leur talent au service de la représentation des succès du socialisme en construction, $\mathrm{y}$ compris de la politique nationale, notamment à l'égard des juifs soviétiques.

3 À l'orée de la guerre, ces photographes sont clairement impliqués dans le projet soviétique et il va leur falloir photographier l'entreprise de destruction inouïe menée par les nazis sur leur propre sol. Ce sont eux qui prendront, dès les premières libérations de 
territoires au début de 1942, les premiers clichés de l'Holocauste, celui qui s'est déroulé dans les ravins soviétiques; avant de suivre l'Armée rouge dans sa reconquête, à partir de 1943, des territoires occidentaux, où se concentraient la grande majorité des juifs vivant en Union soviétique au moment de l'invasion allemande; puis son entrée en Europe orientale, libérant les camps de concentration et d'extermination polonais, avant de triompher à Berlin.

David Shneer brosse ensuite rapidement les sombres années d'après-guerre, où beaucoup de ces photojournalistes se retrouvent sans travail, jusqu'à un nouvel essor à partir de la fin des années 1950, lorsque la commémoration de la guerre offre une nouvelle carrière à certains de leurs clichés, voire, dans le cas d'un Haldej, le succès après 1991 d'une production dans laquelle l'Occident se plait à retrouver l'identité juive.

Or, c'est bien la question de l'identité juive qui est au cœur de la problématique de l'ouvrage. Que veut dire être juif pour ces photographes soviétiques? David Shneer pose d'emblée que cette identité juive varie selon les individus, selon les périodes de leur vie, selon les interlocuteurs auxquels ils s'adressent, mais elle est, selon lui, indéniable, en particulier lorsque le photographe se fait le témoin du massacre de juifs soviétiques. Et pourtant, son étude minutieuse des photos de l'Holocauste, tel que celui-ci se dévoile au fur et à mesure de la progression de l'Armée rouge, montre plutôt une adéquation de ces photographes avec le discours officiel soviétique qui tend à l'universalisation des victimes des atrocités nazies. L'utilisation de sources retrouvées dans plusieurs pays, dont une partie - chose extrêmement rare en histoire soviétique - provient d'archives privées, permet un exposé subtil montrant à la fois les photos prises par le photographe, celles qui ont été sélectionnées pour publication, et parfois, trouvailles de l'historien, ce que le photographe a écrit pour lui, dans son carnet de terrain, sur ce dont il était le témoin. Un chapitre spécifique sur la presse en yiddish (ou plus précisément sur le journal du Comité juif antifasciste Eynigkayt) permet de montrer un traitement de l'Holocauste différent de la presse en langue russe, y compris du point de vue de la publication de photos. David Shneer combine l'étude de la photographie et du texte qui l'accompagne : légende, article, afin d'établir si l'identité juive des victimes est précisée, occultée, voire suggérée.

Cette analyse peut parfois faire sourciller. Prenons l'exemple des photos prises à Kerš en janvier 1942 par plusieurs journalistes juifs soviétiques - qui sont pour Shneer les premières photos publiées de libération de l'Holocauste (à distinguer des photos prises sur les soldats allemands morts ou capturés, représentant les massacres perpétrés en Pologne dès 1939), et donc bien avant les photos des camps polonais. L'auteur montre bien que l'identité juive des victimes n'est jamais signalée, ni dans les légendes ou les articles ni dans le message implicite de ces photos. Après la publication des photos de Mark Redkin - montrant des cadavres le long d'un fossé antichar, sans indication de leur identité juive, dans un numéro de la revue phare du photojournalisme, Ogonek, en février 1942 -, les photos de Dmitrij Bal'termanc publiées en mars dans la même revue montrent des cadavres, mais aussi des vivants qui sont, eux, explicitement identifiés comme Slaves. Il s'agit de vieilles femmes à la recherche des leurs dans un paysage d'horreur : P.I. Ivanova qui vient de retrouver son époux, V.S. Tereščenko remuant des corps pour retrouver celui de son mari. La juxtaposition d'une photo d'un cadavre identifié comme un certain Kogan ne suggère pas forcément l'existence de couples interethniques, ni le fait que la partie slave de la population de la ville a été épargnée, contrairement à ce qu'avance David Shneer, mais va dans le sens du texte qui accompagne la photo: ici 
furent assassinés des habitants de plusieurs nationalités, dont des juifs. Cette idée est largement colportée par juifs et non-juifs dans les publications en langue russe. Une autre photo de Kerš, prise par Bal'termanc, connaît une seconde vie dans les années 1960 et est à ce jour une des plus célèbres représentations de la guerre en URSS - et non spécifiquement de l'Holocauste des juifs -, puisqu'elle représente P.I. Ivanova face au cadavre de son époux, sur un sol lunaire jonché de cadavres.

7 L'histoire des photos de Kerš montre donc la participation des photographes juifs soviétiques et, au-delà, des juifs soviétiques qui prirent leur plume, au silence sur le sort spécifique des juifs soviétiques en territoire occupé. Comme l'écrit lui-même David Shneer, la presse soviétique a pour consigne, dès le début de la guerre, de montrer à la fois l'héroïsme soviétique et les atrocités commises par les nazis. Or, si le texte qui les accompagne ne le précise pas, comment reconnaître dans les photos, publiées ou non, prises par ces photographes soviétiques juifs, l'identité juive des victimes? En d'autres termes, qu'est ce qui pourrait distinguer les photos d'atrocités selon l'ethnicité des victimes? David Shneer ne répond pas à cette question, d'autant que, s'il précise à plusieurs reprises que le nombre de victimes soviétiques non combattantes excède de beaucoup les seules victimes de l'Holocauste, il ne parle jamais des atrocités concernant des non-juifs, à l'exception, involontairement, de la tragédie de Leonovo, sans doute un massacre de villageois dont les maisons furent brûlées et que Shneer range dans les victimes de l'Holocauste (p. 127). Autre point crucial, en quoi les photos prises par les photographes juifs de victimes juives diffèrent-elles des photos prises par des photographes non juifs? Shneer suggére que la façon de photographier des reporters et les photos trouvées dans les dossiers de la Commission d'enquête soviétique généralement non attribuées donc sans indication de la nationalité, au sens soviétique du terme, de leurs auteurs - tendent à se confondre, mais aucune étude systématique ne vient répondre à cette question pourtant fondamentale dans la problématique du livre.

Le traitement de l'Holocauste par la presse soviétique en langue yiddish montre la même complexité et la même ambiguïté, même si son approche est différente. L'héroïsme juif est ainsi un thème fondamental de Eynigkayt, suivi des atrocités commises par les nazis. L'identité juive des victimes y est clairement signalée, quoique Shneer donne aussi des exemples d'universalisation des victimes. Cependant, du point de vue du sujet central du livre - la photographie -, les conclusions ne sont pas évidentes. Shneer précise ainsi que les photographes ne prenaient pas de clichés spécifiquement destinés à cette presse, même si des photos identifiant clairement des juifs y sont publiées. Il évoque notamment celle d'un couple de survivants juifs du ghetto de Budapest photographié par Haldej au début de 1945, et publiée exclusivement par Eynigkayt, avant de connaître une formidable notoriété après 1991. Surtout, Eynigkayt a publié très peu de photos: aucune notamment de Majdanek, dont la libération a pourtant été abondamment couverte par la presse soviétique en langue russe. Shneer nous soumet deux hypothèses: absence de moyens matériels et volonté de ne pas judaïser Majdanek. De façon intéressante, il relie cette dernière préoccupation à la réticence comparable des journalistes juifs américains.

9 De fait, une autre question posée par l'ouvrage reste en grande partie ouverte. À quel stade et sous quelle forme intervenait la censure? On connaît le travail de réécriture pour gommer l'identité juive des victimes dans la plupart des rapports publiés de la Commission d'enquête soviétique. Cependant, l'auteur n'a pas trouvé de consignes particulières en ce qui concerne les photographes sur ce sujet (il a retrouvé dans les archives de Bal'termanc des consignes envoyées par sa rédaction, mais qui concernent la 
couverture de la bataille de Stalingrad et non les photos d'atrocités). Quelle est donc la part d'autocensure? L'affirmation, à la suite de Denise Youngblood et de bien d'autres, que la guerre fut un " petit oasis de liberté $»^{1}$ en la matière, n'est en rien confirmé par le propos de l'ouvrage: tout indique un fort contrôle (ne serait-ce que par la durée généralement assez longue entre la prise du cliché et sa publication), même s'il est difficile d'en établir précisément les étapes et les formes. David Shneer aurait même pu aller plus loin dans cette direction : rien n'est dit, pour ne prendre que cet exemple, du travail de mise en scène accompli avec les survivants d'Auschwitz.

L'ouvrage n'apporte pas toujours de réponses définitives - faute en partie d'une méthodologie plus rigoureuse qui aurait pris en compte systématiquement la production des photographes juifs et non juifs, ainsi que les atrocités ne concernant pas au premier chef les juifs d'Union soviétique; le tout dans un souci comparatif qui aurait démontré la spécificité juive des photographes plutôt que de la poser comme un fait donné, certes variable d'un individu à l'autre. Il faut néanmoins saluer la parution de cette contribution, à la fois riche et nuancée, à un questionnement très actuel sur la représentation de la Grande Guerre patriotique par les autorités soviétiques.

\section{NOTES}

1. Denise Youngblood, War Films: On the Cinema Front, 1914-2005, Lawrence, KS : University Press of Kansas, 2007, cité p. 91. 